\title{
K-space model of motion artifacts in synthetic transmit aperture ultrasound imaging
}

\author{
Nikolov, Svetoslav; Jensen, Jørgen Arendt
}

Published in:

IEEE Symposium on Ultrasonics, 2003

Link to article, DOI:

10.1109/ULTSYM.2003.1293268

Publication date:

2003

Document Version

Publisher's PDF, also known as Version of record

Link back to DTU Orbit

Citation (APA):

Nikolov, S., \& Jensen, J. A. (2003). K-space model of motion artifacts in synthetic transmit aperture ultrasound imaging. In IEEE Symposium on Ultrasonics, 2003 (pp. 1824-1828). IEEE.

https://doi.org/10.1109/ULTSYM.2003.1293268

\section{General rights}

Copyright and moral rights for the publications made accessible in the public portal are retained by the authors and/or other copyright owners and it is a condition of accessing publications that users recognise and abide by the legal requirements associated with these rights.

- Users may download and print one copy of any publication from the public portal for the purpose of private study or research.

- You may not further distribute the material or use it for any profit-making activity or commercial gain

- You may freely distribute the URL identifying the publication in the public portal

If you believe that this document breaches copyright please contact us providing details, and we will remove access to the work immediately and investigate your claim. 


\title{
K-Space Model of Motion Artifacts in Synthetic TRANSMIT ULTRASOUND IMAGING
}

\author{
Svetoslav Ivanov Nikolov and Jørgen Arendt Jensen \\ Center for Fast Ultrasound Imaging, Bldg 348, Ørsted•DTU, Technical University of Denmark \\ DK-2800 Lyngby, Denmark
}

\begin{abstract}
Synthetic transmit aperture (STA) imaging gives the possibility to acquire an image with only few emissions and is appealing for 3D ultrasound imaging. Even though the number of emissions is low, the change in position of the scatterers prohibits the coherent summations of ultrasound echoes and leads to distortions in the image. In order to develop motion compensation and/or velocity estimation algorithms a thorough and intuitive understanding of the nature of motion artifacts is needed.

This paper proposes a simple 2D broad band model for STA images, based on the acquisition procedure and the beamformation algorithm. In STA imaging a single element transmits a cylindrical wave. All elements are used in receive, and by applying different delays a low resolution image (LRI) is beamformed. A Fourier relation exists between the aperture function and all points in the beamformed LRI. This relation is used to develop an approximation of the point spread function (PSF) of a LRI. It is shown that the PSF of LRIs obtained by transmitting with different elements can be viewed as rotated versions of each other. Summing several LRIs gives a high resolution image.

The model approximates the PSF of a high resolution image as a sum of rotated PSFs of a single LRI. The approximation is validated with a Field II simulation.

The model predicts and explains the motion artifacts, and gives an intuitive feeling of what would happen for different velocities.
\end{abstract}

\section{INTRODUCTION}

Synthetic aperture (SA) imaging has the advantage over the conventional phased or linear array ultrasound imaging in that it yields higher frame rates [1] is easily extendible to 3D [2] and generates images which are perfectly focused both in transmit and receive. One frame is, however, acquired over a number of emissions, and if the scatterers move, then their echoes do not arrive at the expected time instance. This prevents the coherent summation of the echoes.

Previously it was shown that images created with the same transmission sequence, and subjected to the same motion suf- fer from the same motion artifacts. The correlation between such images is high and it is possible to estimate the flow with high precision $[1,3]$.

In this paper a model for the motion artifacts is presented. This model explains why images acquired with the same transmission sequence exhibit high-correlation. The model also makes it easy to predict the shape of the point-spreadfunction (PSF) in case of motion. The purpose of this work is to develop an intuitive feeling in the reader of what the nature of motion artifacts is, and how one can design new motion compensation or flow-estimation algorithms.

The model is based on frequency domain approximations which are introduced in Sec. II. These approximations are used in Sec. III to illustrate the forming of an ultrasound image. Finally the application of the model is discussed in Sec. V.

\section{SOME FOURIER RELATIONS}

SA ultrasound imaging is made by sending out de-focused waves. The defocusing decreases the pressure and non-linear effects can be neglected so that a linear system description is adequate. The pulse-echo response $p_{r}(\vec{x}, t)$ of a single scatterer at location $\vec{x}_{1}$ is given by [4]

$$
p_{t / r}(\vec{x}, t)=v_{t / r}(t) \underset{t}{*} \delta\left(\vec{x}-\vec{x}_{1}\right) \underset{x}{*} h_{t / r}(\vec{x}, t),
$$

where $v_{t / r}$ is a term accounting for transducer excitation and impulse response of the scanner, and $h_{t / r}(\vec{x}, t)$ is the spatial pulse-echo impulse response accounting for the wave propagation and diffraction. The symbols $\underset{t}{*}$ and $\underset{x}{*}$ denote convolution in time and space, respectively. For SA focusing, the image is focused at all points. We will assume, that in the neighborhood of the focal point, the spatial impulse response does not change, which makes it possible to describe it as a separable function. The spatial impulse response for the transmitting aperture is:

$$
h_{t}(\theta, t)=h_{p 1}(\theta) \cdot h_{p 2}(t)
$$




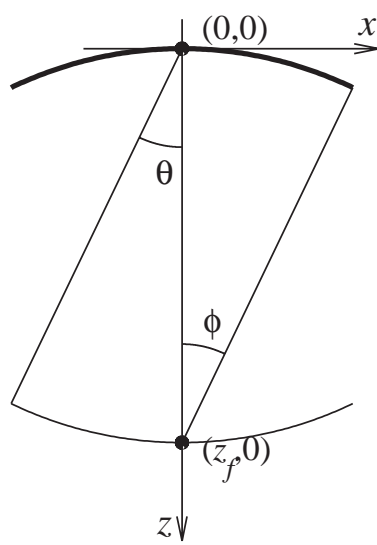

(a)

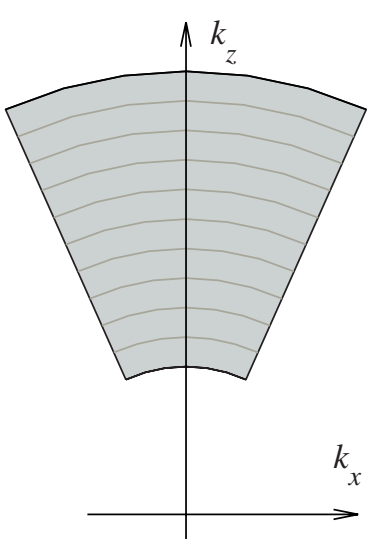

(b)
Figure 1: The geometry for the Fraunhofer approximation of the radiation field at the focal point. Plot (a) illustrates the geometry, and (b) illustrates the spectrum of the point spread function.

The radiated pressure field $p_{t}\left(\sin \theta, t ; z_{f}\right)$ at fixed depth $z_{f}$ $\left(x_{f}=0\right)$ becomes:

$$
\begin{gathered}
p_{t}\left(\sin \theta, t ; z_{f}\right)=\mathrm{A}_{t}\left(\sin \theta ; z_{f}\right) \cdot v_{p}(t), \\
v_{t}(t)=g(t) \underset{t}{*} h_{t}(t) * h_{t} h_{p 2}(t),
\end{gathered}
$$

where $A_{t}\left(\sin \theta ; z_{f}\right)$ is the radiation pattern of the transducer when a delta function is used as an excitation, $g(t)$ is the transmitted pulse, and $h_{t}(t)$ is electro-mechanical impulse response. The relation is valid over an arc in the neighbourhood of $\vec{x}_{f}$, and is therefore given as a function of the azimuth angle $\theta$. The radiation pattern $\mathrm{A}_{p}\left(\sin \theta ; z_{f}\right)$ can be found from:

$$
\mathrm{A}_{p}\left(\sin \theta ; z_{f}\right)=\int_{-\infty}^{\infty} A_{p}\left(\omega, \sin \theta ; Z_{f}\right) d \omega,
$$

where $A_{p}\left(\omega, \sin \theta ; z_{f}\right)$ is the radiation pattern for a single frequency $\omega$. One can use the Fraunhofer approximation to describe it. The Fraunhofer approximation is valid in two cases: (a) the far field, and (b) the focal point in the case of a focused transducer $[5,6]$. It basically states that the radiated field and the apodization functions are related through the Fourier transform ${ }^{1}$ :

$$
\mathrm{A}\left(\omega, \sin \theta ; z_{f}\right)=\mathcal{F}^{-1}\{a(k \sin \phi ; 0)\},
$$

where $\omega$ is the angular frequency, $k=\omega / c$ is the wavenumber, $\theta$ and $\phi$ are angles as shown in Fig. 1(a). The figure shows the 2-D case of a transducer which is focused at

\footnotetext{
${ }^{1}$ The exact expressions can be taken from one of the references Ref. [1, $5,6]$
}

a fixed point $\vec{x}_{f}=\left(0, z_{f}\right)$. The center of the transducer coincides with the origin of the coordinate system $(0,0)$. The angle $\theta$ is measured relative to the $z$ axis (depth), and will be called "azimuth angle". The radiated field is studied along an arc passing through the focal point $\left(0, z_{f}\right)$. The two arcs - the surface of the transducer and the line over which the field is studied - are confocal $[1,6]$.

Taking the Fourier transform of the PSFs gives the frequency response of the system, which is illustrated in Fig. 1 (b). The two spatial frequencies $k_{x}$ and $k_{z}$ are related through:

$$
k^{2}=k_{x}^{2}+k_{z}^{2},
$$

which is indicated by the arcs drawn within the spectrum. The arcs are within a limited angle span due to the finite size of the transducer. Most transducers acts as bandpass filters $k \in\left[k_{1}, k_{2}\right]$, and the shape of the amplitude spectrum has a bell-like shape. When viewed as an image, the 2-D spectrum appears like a "tear-drop".

Since $p_{t}\left(\sin \theta, t ; z_{f}\right)$ is a separable function, then the twodimensional Fourier transform of (3) gives also a separable function:

$$
\begin{gathered}
P_{t}\left(k_{x}, \omega ; z_{f}\right)=\mathcal{F}\left\{\mathrm{A}_{t}\left(\sin \theta ; z_{f}\right)\right\} \cdot \mathcal{F}\left\{v_{t}(t)\right\} \\
P_{t}\left(k_{x}, \omega ; z_{f}\right)=a_{t}(k \sin \phi ; 0) \cdot V_{t}(\omega),
\end{gathered}
$$

It can be seen that the bandwidth of $k_{x}$ is proportional to the size of the aperture.

\section{Model of THE ACQUisition Process}

Synthetic transmit aperture (STA) ultrasound imaging is done by transmitting with a single element and receiving with all elements. An image can be formed by using a delay-and-sum beamforming. The image is formed as:

$$
\begin{aligned}
& \mathbf{H}(\vec{x})=\sum_{i=1}^{N} \sum_{j=1}^{N} a_{i j}(\vec{x}) r(t(\vec{x})) \\
& t(\vec{x})=\frac{1}{c}\left(\left|\vec{x}-\vec{x}_{i}\right|+\left|\vec{x}-\vec{x}_{j}\right|\right),
\end{aligned}
$$

where $r_{i j}(t)$ is the signal received by element $j$ after transmiting with element $i, \vec{x}_{i}$ and $\vec{x}_{j}$ are their spatial coordinates, and $\vec{x}$ are the coordinates of the points from the image, $a_{i j}$ are the apodization coefficients, and $N$ is the number of elements. The process can be split in two steps:

$$
\begin{gathered}
\mathbf{L}_{i}(\vec{x})=\sum_{j=1}^{N} a_{j}(\vec{x}) r_{i j}(t(\vec{x})) \\
\mathbf{H}(\vec{x})=\sum_{i=1}^{N} a_{i}(\vec{x}) \mathbf{L}_{i}(\vec{x}),
\end{gathered}
$$

$\mathbf{L}_{i}(\vec{x})$ is an image in itself, and it has a low resolution, since the beamforming has been done only in receive. The time at 

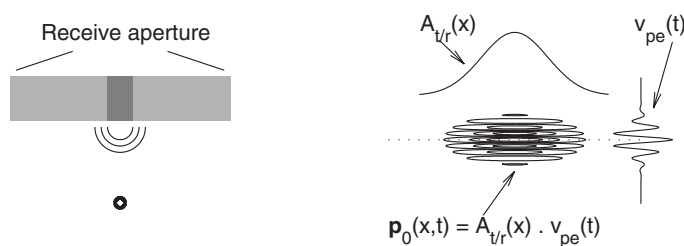

(a)

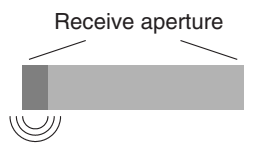

-

(b)
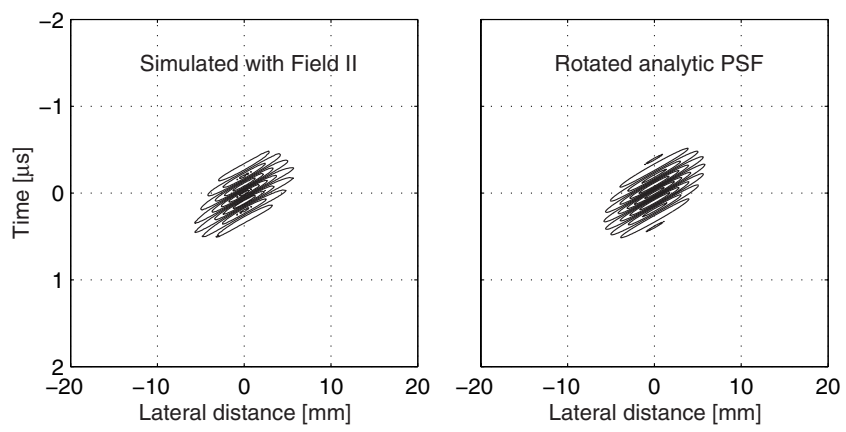

(c)

Figure 2: Comparison between the PSF created with Field II and an analytically created and rotated PSF

which the signal is taken is dynamically calculated for every point, and therefore the Fourier relation from Sec. II is valid for the receive aperture for all points in the image. In transmit only a single element is used, the points of the image are in the far field of the transmitting elements, and therefore the Fourier relation is valid for all points in the image and the transmit aperture. The pulse/echo radiation pattern can be found as:

$$
\mathrm{A}_{t / r}\left(\sin \theta ; z_{f}\right)=\mathrm{A}_{t}\left(\sin \theta ; z_{f}\right) \cdot \mathrm{A}_{r}\left(\sin \theta ; z_{f}\right),
$$

where $A_{t}$ and $A_{r}$ are the radiation patterns of the transmit and receive apertures, respectively. The angular spectrum of the point spread function of the low resolution image can be approximated as follows:

$$
P_{t / r}\left(k_{x}, \omega ; z_{f}\right)=\underbrace{a_{t}(k \sin \phi) * a_{r}(k \sin \phi)}_{a_{t / r}(k \sin \phi)} \cdot \mathcal{F}\left\{v_{t / r}(t)\right\},
$$

where $a_{t}(k \sin \phi ; 0)$ and $a_{r}(k \sin \phi ; 0)$ are the apodization functions of the transmit and receive apertures, respectively, and $a_{t / r}(\sin \phi)$ is known as the effective aperture. The effective aperture is a fictive aperture that would have a one-way radiation pattern as the pulse-echo radiation pattern of the system. We will model the transmit element as a point source: $a_{t}\left(k \sin \phi_{0}\right)=\delta\left(k \sin \phi-k \sin \phi_{0}\right)$. The point spread function can be found by taking the inverse Fourier transform of $P_{t / r}\left(k_{x}, \omega ; z_{f}\right)$ :

$$
\mathbf{p}\left(\sin \theta, t ; z_{f}\right)=\mathcal{F}^{-1}\left\{a_{t}(k \sin \phi) *_{x} a_{r}(k \sin \phi)\right\} \cdot v_{t / r}(t)
$$

Notice that a linear array is used. If the position of the transmit element changes, then a different delay is used to accomodate for the propagation path. This corresponds to moving a point source along the arc defining the array in Fig. 1 (a). Changing the position of the transmitting element corresponds to changing the position of the whole setup:

$$
\begin{aligned}
a_{t / r}(u-\Delta u) & =a_{t}(u-\Delta u) \underset{x}{*} a_{r}(u) \\
& =a_{t}(u) \underset{x}{*} a_{t}(u-\Delta u) \\
& =a_{t}(u-\Delta u / 2) a_{r}(u-\Delta u / 2),
\end{aligned}
$$

where $u=k \sin \phi$. In other words, the spectrum of the low resolution image $\mathbf{L}(\vec{x})$ is being rotated (see Fig. 1(b)) at an angle

$$
\beta \approx \arcsin \frac{\Delta x}{z_{f}},
$$

where $\Delta x$ is the distance between the center of the transmitting element and the center of the array. A rotation of the 2-D spectrum corresponds to a rotation of the 2-D image. This operation can be expressed as:

$$
\mathbf{p}^{\prime}\left(x, z ; \vec{x}_{f}\right)=\mathcal{R}\left[\beta ; \vec{x}_{f}\right]\left\{\mathbf{p}\left(x, z ; \vec{x}_{f}\right)\right\},
$$

where $\mathcal{R}\left[\beta ; \vec{x}_{f}\right]$ means "rotation at an angle $\beta$ around the coordinates $\vec{x}_{f}=\left(x_{f}, z_{f}\right)$ ". The rotation is given as:

$$
\begin{aligned}
\mathbf{p}\left(x^{\prime}, z^{\prime} ; z_{f}\right) & =\mathbf{p}\left(x, z ; z_{f}\right) \\
{\left[\begin{array}{l}
x^{\prime} \\
z^{\prime}
\end{array}\right] } & =\left[\begin{array}{cc}
\sin \beta & \cos \beta \\
\cos \beta & -\sin \beta
\end{array}\right]\left[\begin{array}{l}
x-x_{f} \\
z-z_{f}
\end{array}\right]+\left[\begin{array}{l}
x_{f} \\
z_{f}
\end{array}\right]
\end{aligned}
$$

This is illustrated in Fig. 2. Fig. 2(a) shows how the point spread function of a low resolution image is approximated when the transmission is done with the central element. The apodization function of the receive aperture $a_{r}(x)$ is chosen to be a Gausian function. The transmit element is assumed to be omnidirectional. The two way radiation pattern $\mathrm{A}_{t / r}(x)$ is equal to $\mathrm{A}_{r}(x)$, and is Gausian function too. The point spread function $\mathbf{p}_{0}(x, t)$ of the low resolution image is parallel to the transducer surface. Fig. 2(b) show the case when the outermost element of a transducer is used in transmit. Its point 


\begin{tabular}{lccr}
\hline \hline Parameter & Notation & value & unit \\
\hline Speed of sound & $c$ & 1540 & $\mathrm{~m} / \mathrm{s}$ \\
Center frequency & $f_{0}$ & 5 & $\mathrm{MHz}$ \\
Sampling frequency & $f_{s}$ & 70 & $\mathrm{MHz}$ \\
Wavelength & $\lambda_{0}$ & 308 & $\mu \mathrm{m}$ \\
Fractional bandwidth & $\mathrm{BW}$ & 66 & $\%$ \\
No elements & $N_{x d c}$ & 65 & - \\
Pitch & $d_{x}$ & 308 & $\mu \mathrm{m}$ \\
\hline \hline
\end{tabular}

Table 1: Simulation parameters used for the simulations.

spread function $\mathbf{p}_{i}(x, t)$ is approximated by rotating $\mathbf{p}_{0}(x, t)$ at an angle $\beta_{i}$ as given in (18):

$$
\beta_{i}=\arcsin \frac{\left(i-\frac{N+1}{2}\right) d_{x}}{2 z_{f}},
$$

where $d_{x}$ is the distance between the centers of two elements, $i \in[1, N]$ is the index of the transmitting element, and $z_{f}$ is the distance to the focal point as shown in Fig. 1.

The final high resolution image (HRI) is the sum of the low resolution images, and so is its point spread function:

$$
\begin{aligned}
\mathbf{P}(x, z ; \vec{x} f) & =\sum_{i=1}^{N} \mathbf{p}_{i}\left(x, z ; \vec{x}_{f}\right) \\
\mathbf{P}(x, z ; \vec{x} f) & =\sum_{i=1}^{N} \mathcal{R}\left[\beta_{i}, \vec{x}_{f}\right]\left\{\mathbf{p}_{0}\left(x, z ; \vec{x}_{f}\right)\right\}
\end{aligned}
$$

\section{MODEL OF THE MOTION ARTIFACTS}

In order to continue the considerations, it will be assumed that the PSF of the low-resolution images does not change for small changes in the axial and lateral positions:

$$
\mathbf{p}_{0}\left(x, z ; \vec{x}_{f}+\Delta \vec{x}\right)=\mathbf{p}_{0}\left(x, z ; \vec{x}_{f}\right)
$$

The point spread function of the point at the new location will merely be a translated version of the PSF at position $\vec{x}_{f}$ :

$$
\mathbf{p}_{i}\left(x, z ; \vec{x}_{f}+\Delta \vec{x}\right)=\mathcal{T}[\Delta \vec{x}]\left\{\mathbf{p}_{i}\left(x, z ; \vec{x}_{f}\right)\right\} .
$$

$\mathcal{T}[\Delta \vec{x}]\left\{\mathbf{p}_{i}(x, z ; \vec{x})\right\}$ is used to denote translation of the PSF:

$$
\begin{gathered}
\mathbf{p}_{i}\left(x^{\prime}, z^{\prime} ; \vec{x}_{f}+\Delta \vec{x}\right)=\mathbf{p}_{i}\left(x, z ; \vec{x}_{f}\right) \\
{\left[\begin{array}{l}
x^{\prime} \\
z^{\prime}
\end{array}\right]=\left[\begin{array}{l}
x \\
z
\end{array}\right]+\left[\begin{array}{l}
\Delta x \\
\Delta z
\end{array}\right] .}
\end{gathered}
$$

Figure 3 shows the comparison between PSFs of highresolution images, obtained without and with motion. The top row displays the imaging situation. The emissions are done consecutively with the outermost elements, first with the leftmost and then with the rightmost one. The positions of the scatterer and the PSFs of the low-resolution images obtained at the first and the second emissions are given in blue and red colors, respectively. The middle row shows the superimposed low resolution images obtained at each of the emissions. The thick lines outline the envelope detected data at -1 and $-20 \mathrm{~dB}$. The thin and pale lines are contours of the raw RF signal, and are presented to show the direction and frequency of the oscillations. The distance which the point scatterer passes between the two positions is chosen to be $\lambda / 4$ at a center frequency $f_{0}$ of $5 \mathrm{MHz}$. The PSFss were obtained using Field II, and the simulation parameters are listed in Table 1 . The bottom row shows contour plots of the envelope detected high-resolution images. The contours are drawn at levels $-6,-10$ and $-20 \mathrm{~dB}$ from the peak value. It can be seen that the axial motion results in a distortion of the PSF. The PSF becomes asymmetric and its maximum shifts away from the central line. The lateral motion introduces only minor change in the PSF. The reason for this is the anisotropy of the PSF - the lateral size is several times larger than the axial one. The process of creating a high-resolution image in the presence of motion becomes:

$$
\left.\mathbf{P}\left(x, z ; \vec{x}_{f}\right)=\sum_{i=1}^{N_{x m t}} \mathcal{T}[i \Delta \vec{x}]\left\{\mathcal{R}\left[\beta_{i} ; \vec{x}_{f}\right)\right]\left\{\mathbf{p}_{0}\left(x, z ; \vec{x}_{f}\right)\right\}\right\},
$$

where $\beta_{i}$ is the angle relate to the transmitting element $i$ via the relation (23).

\section{DIscussion}

$K$-space can be employed to analyze a number of problems in medical ultrasound. As pointed out in [5] simple graphical methods are useful for the intuitive understanding of the image formation, and can be used also as an excellent teaching tool. The main contribution of this paper is that the geometrical model is given in 2-D rather than 1-D (most other papers consider only $\mathrm{A}(x)$, rather than $\mathbf{p}(x, z)$ ). This makes it possible to easily explain some effects of motion. For example, Hazard and Lockwood [7] make the observation that if an image is made using three emissions, the peak of the PSF is split in two, and that the side-lobe level rises with about $5 \mathrm{~dB}$. Looking at Fig. 3 one can see that if there were three low resolution images, then the resulting two sums will be centered around two points, rather than one. These two points will not coincide with the peak amplitude of any of the low resolution images, and the ratio between the sum and side lobes will be lower.

This model helps explain why it is possible to estimate the velocity using synthetic aperture imaging. Figure 4 illustrates the building of high resolution images at every emission. The low resolution images $\mathbf{L}$ obtained after transmitting with the same element are translated versions of each other. The PSFs 

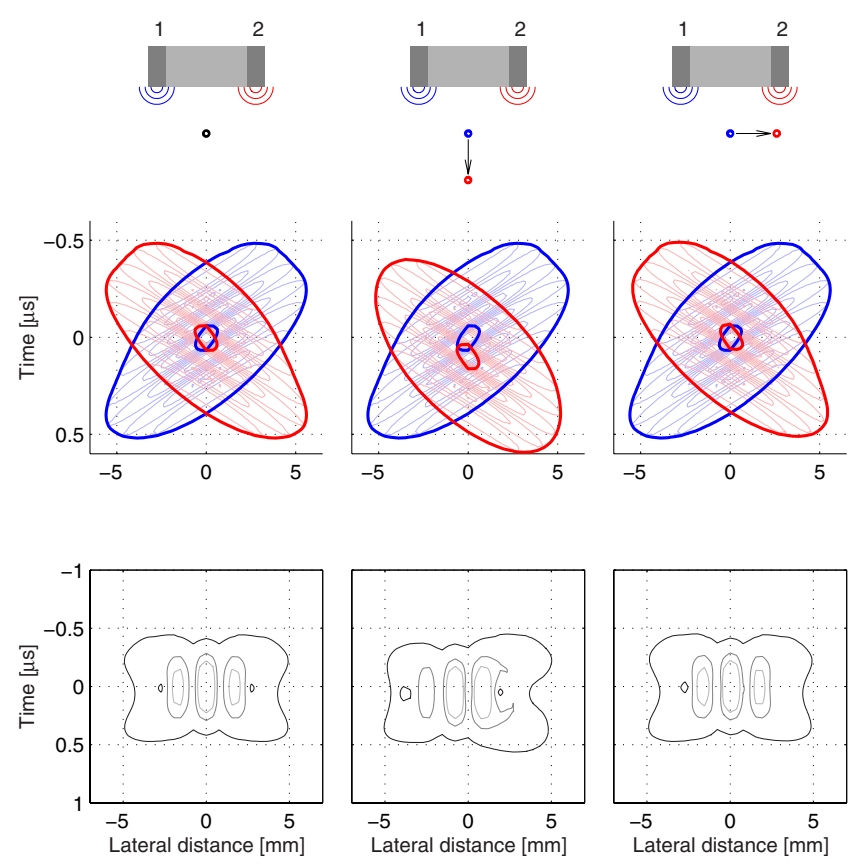

Figure 3: Making a high-resolution image with only two emissions.

of the high resolution images obtained using the same transmit sequence are also translated versions of each other. In

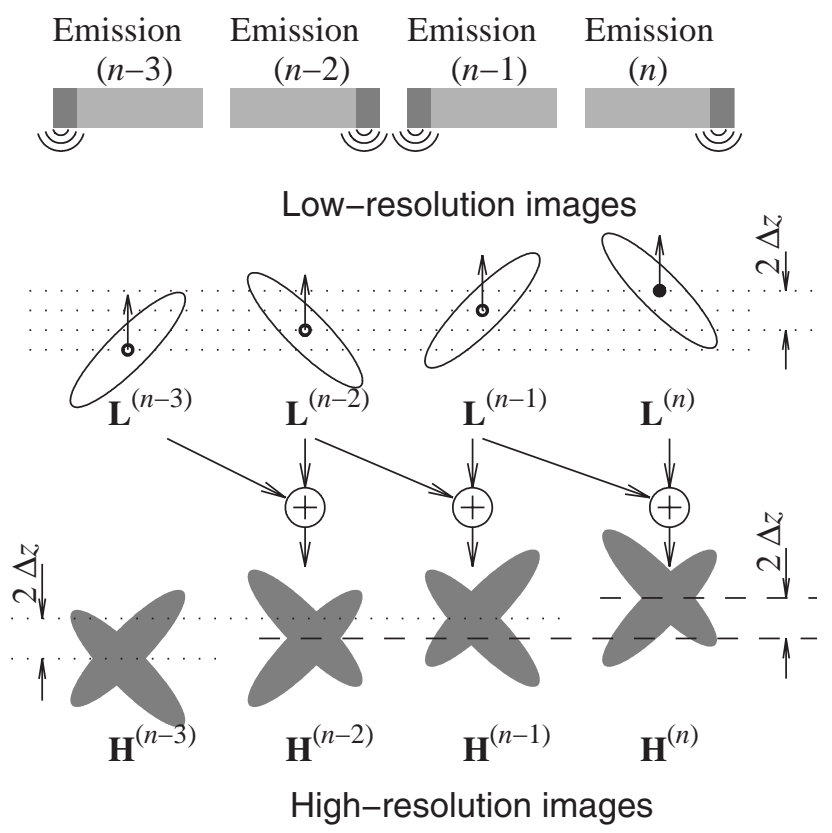

Figure 4: High resolution images made at consecutive emissions. Only two of the elements are used in transmit.
Fig. 4, there are two pairs of images, which are created with the same transmit sequence : $\mathbf{H}^{(n-3)}$ and $\mathbf{H}^{(n-1)}$, and $\mathbf{H}^{(n-2)}$ and $\mathbf{H}^{(n)}$. If a group of scatterers has moved at the distance $\Delta z$, then the resulting signal segements will be a translated versions, and this translation can be estimated as described in $[1,3]$.

The model can be used in designing motion compensation algorithms. From (28), it can be seen that HRI can be expressed as a sum of translated low-resolution images. If one first estimates the vectorial flow [8], then one should translate back (pick the right samples) from the low resolution images and sum them to get a motion compensated high-resolution image.

\section{CONCLUSION}

This paper has presented a conceptual tool which allows the analysis in $k$-space of synthetic aperture imaging. The developed simple geometric model makes it possible to rapidly identify and qualify phenomena related to the effects of motion, and helps understand how an ultrasound image is actually built.

\section{REFERENCES}

[1] S. I. Nikolov. Synthetic aperture tissue and flow ultrasound imaging. PhD thesis, Ørsted•DTU, Technical University of Denmark, 2800, Lyngby, Denmark, 2001.

[2] S. I. Nikolov, R. Dufait, A. Schoisswohl, and J. A. Jensen. Three-dimensional real-time synthetic aperture imaging using a rotating phased array transducer. In Proc. IEEE Ultrason. Symp., pages 1545-1548, 2002.

[3] S. I. Nikolov and J. A. Jensen. In-vivo synthetic aperture flow imaging in medical ultrasound. IEEE Trans. Ultrason., Ferroelec., Freq. Contr., pages 848-856, 2003.

[4] J. A. Jensen. Linear description of ultrasound imaging systems, notes for the international summer school on advanced ultrasound imaging. Technical report, Department of Information Technology, DTU, 1999.

[5] W. F. Walker and G. E. Trahey. The application of kspace in pulse echo ultrasound. IEEE Trans. Ultrason., Ferroelec., Freq. Contr., 45:541-558, 1998.

[6] J. W. Goodman. Introduction to Fourier optics. McGraw Hill Inc., New York, second edition edition, 1996.

[7] C. R. Hazard and G. R. Lockwood. Effects of motion artifacts on a synthetic aperture beamformer for real-time 3D ultrasound. In Proc. IEEE Ultrason. Symp., pages 1221-1224, 1999.

[8] J. A. Jensen and S. I. Nikolov. Transverse flow imaging using synthetic aperture directional beamforming. In Proc. IEEE Ultrason. Symp., pages 1488-1492, 2002. 